\title{
Depressão Psicótica no Idoso: Desafio Diagnóstico e Papel da Eletroconvulsivoterapia
}

Psychotic Depression in the Elderly: Diagnostic Challenge and Role of Electroconvulsive Therapy

Ricardo Coentre, Amílcar Santos, Joana Lopes, Maria João Gracias, Miguel Talina

\section{RESUMO}

Os autores descrevem o caso clínico de um doente com 69 anos com depressão psicótica e sintomas físicos exuberantes. Dados os sintomas físicos significativos e o escalão etário foi efetuada avaliação médica que permitiu excluir doença orgânica. Apesar da terapêutica psicofarmacológica realizada, o doente não apresentou melhoria clínica significativa. Foi efetuada terapêutica eletroconvulsiva ainda na fase aguda, e por recidiva sob terapêutica antidepressiva, também de continuação e de manutenção. Atingiu-se assim uma remissão total do quadro clínico e de funcionamento, tendo o doente retomado a sua atividade profissional. O relato deste caso justifica-se pela sua apresentação peculiar e pelo benefício alcançado com a realização da eletroconvulsivoterapia (aguda e de continuação/manutenção), associado a terapêutica psicofarmacológica que garantiu uma remissão clínica significativa.

PALAVRAS-CHAVE: Depressão; Eletroconvulsivoterapia; Idoso

\section{ABSTRACT}

The authors describe the clinical case of a 69-year-old patient with psychotic depression and exuberant physical symptoms. Because of the significant physical symptoms and the age group, a medical assessment was made that allowed organic disease to be excluded. Despite the psychopharmacological therapy, the patient did not present any significant clinical improvement. Electroconvulsive therapy was conducted in the acute phase, and by relapse under anti-depressive therapy, also on-going and for maintenance. A total remission of the clinical and functional situation was achieved and the patient restarted professional activity. The report of this case is justified by its peculiar presentation and the benefit that was achieved with electroconvulsive therapy (acute and continuation/maintenance), associated with the psychopharmacological therapy that ensured a significant clinical remission.

KEYWORDS: Depression; Electroconvulsive Therapy; Aged 


\section{INTRODUÇÃO}

A depressão é uma doença de elevada prevalência, sendo que a sua incidência tem vindo a aumentar. $\mathrm{O}$ estudo epidemiológico de doença mental em Portugal encontrou uma prevalência de vida de 16,7\% para a perturbação depressiva major e de 1,4\% para a distimia (perturbação depressiva persistente). ${ }^{1}$ A etiopatogénese da perturbação depressiva é multifatorial. Os fatores genéticos contribuem para uma vulnerabilidade para a doença, que requerem a existência de fatores não genéticos para que esta se manifeste. ${ }^{2}$ Os fatores não genéticos incluem uso de substâncias, características de personalidade (ex.: neuroticismo, baixa autoestima) e eventos adversos (ex.: perda parental, trauma). Fatores psicológicos e sociais podem também contribuir para a etiologia da depressão. Todos estes fatores induzem alterações na estrutura e funções cerebrais, incluindo diversos neurotransmissores, eixo hipotálamo-hipófise-supra-renal, córtex pré-frontal e ritmos circadianos que condicionam a depressão. ${ }^{3}$

A depressão na população idosa é um problema significativo e que está associada a elevada comorbilidade, alteração da autonomia, uso excessivo de cuidados de saúde e aumento da mortalidade. ${ }^{4} \bigcirc$ aumento do número de pessoas idosas eleva a frequência de algumas doenças neuropsiquiátricas crónicas, entre elas a depressão. Para além dos fatores de risco referidos para a depressão na população em geral, no idoso acrescem outros como isolamento social, viuvez, divórcio ou separação, baixo nível socioeconómico, comorbilidade médica em geral, defeitos cognitivos e de funcionamento. ${ }^{5} \bigcirc$ diagnóstico diferencial da depressão no idoso é desafiante por múltiplos aspetos, como doença médica concomitante, sintomas de outras doenças que se sobrepõem aos da depressão (fadiga, perda de apetite, queixas de memória etc.), queixas físicas importantes, dificuldades na comunicação devido a défices sensoriais, estigma associado à doença mental, etc. O tratamento da depressão no idoso, tal como noutros grupos etários, inclui psicoterapia, psicofarmacologia e/ou eletroconvulsivoterapia. ${ }^{6} \mathrm{Na}$ escolha da terapêutica antidepressiva, particular atenção deve ser dada às comorbilidades médicas e à polifarmácia, frequentes no doente idoso. A eletroconvulsivoterapia permanece uma importante opção terapêutica nos idosos, fundamentalmente pela sua segurança e eficácia. Está indicada nos casos que não responderam à terapêutica antidepressiva, naqueles com depressão grave e em que existe risco para a vida do doente com necessidade de resposta terapêutica mais rápida.?

\section{CASO CLÍNICO}

Doente do sexo masculino, de 69 anos de idade, leucodérmico, casado, referenciado à consulta de Psiquiatria em abril de 2014, por quadro com cerca de seis meses de evo- lução de emagrecimento, obstipação acentuada, insónia inicial e ansiedade psíquica. À observação, apresentava-se vígil, colaborante, orientado no espaço e no tempo, alo e autopsiquicamente. A memória e a capacidade de raciocínio estavam conservadas, todavia tinha cessado o trabalho na área da contabilidade onde se ocupava. $\bigcirc$ humor era depressivo. $\bigcirc$ doente manifestava ideias delirantes hipocondríacas, afirmando que tinha uma doença grave, provavelmente gastrointestinal, que justificava o seu emagrecimento e obstipação acentuadas. Apresentava ainda ideação delirante de ruína, referindo que o seu rendimento económico atual, sem que tivesse havido alterações significativas, não permitia adquirir os bens essenciais à sua vida. Não apresentava consciência mórbida significativa para o seu estado clínico.

Nos antecedentes pessoais não apresentava história psiquiátrica significativa, sendo que estava medicado há três meses com bromazepam, 3 mg ao pequeno-almoço e zolpidem, 10 mg ao deitar, ambos prescritos pelo seu Médico de Família. Destacava-se também a hipertensão arterial medicada com irbesartan/hidroclorotiazida 150/12,5 mg por dia e controlada. Apresentava história familiar de suicídio (tia materna aos 40 anos de idade) de causa desconhecida.

Dada a gravidade do quadro clínico foi decidido o seu internamento imediato no Serviço de Psiquiatria, com a hipótese diagnóstica de perturbação depressiva major, episódio único grave com sintomas psicóticos, sendo necessário excluir causa orgânica para a sua sintomatologia, nomeadamente doença neoplásica gastrointestinal.

Durante o internamento realizou avaliação clínica laboratorial, que revelou ligeira anemia microcítica (hemoglobina: 12,4 g/dL; hematócrito: 37,4\%; volume globular médio: 78,9fL). Restantes valores analíticos sem alterações, incluindo função tiroideia, ácido fólico, vitamina B12 e serologias para sífilis, VIH 1 e 2. O doente realizou tomografia computorizada crânio-encefálica que revelou "ausência de alterações da densidade ou da morfologia do parênquima encefálico, em particular sugestivas de lesão vascular ou ocupando espaço, supra ou infratentorial". A ressonância magnética crânio-encefálica não mostrou também "significativas alterações de sinal subcortical, sendo normais as dimensões dos ventrículos laterais, do $3^{\circ}$ e $4^{\circ}$ ventrículos sendo também adequadas e de acordo com o esperado no grupo etário, a volumetria das cisternas da base e da fossa posterior, sem assimetrias focais".

Como terapêtica prescreveu-se venlafaxina, que foi titulada até 225 mg por dia, mirtazapina 45 mg ao deitar e risperidona 2 mg ao deitar. $\bigcirc$ doente não apresentou melhoria clínica significativa, mantendo ideias delirantes 
hipocondríacas e sintomas físicos, como a obstipação marcada. Durante o internamento iniciou ainda recusa alimentar. Realizou colonoscopia que mostrou "duas angiodisplasias do cego, uma das quais com cerca de $18 \mathrm{~mm}$ e outra com 6 mm, sem evidência de hemorragia. Pólipo pediculado com 8 mm, no cólon descendente, removido com ansa diatérmica, sem intercorrências, sem outras alterações do lúmen ou da mucosa". O exame anatomopatológico mostrou tratar-se de "pólipo séssil com adenoma tubular com displasia de baixo grau, totalmente excisado".

Apesar da terapêutica psicofarmacológica, o doente não apresentou melhoria clínica significativa ao fim de cerca de 6 semanas de internamento, sendo que teve inclusivamente que ser submetido a entubação naso-gástrica para garantir a sua alimentação. Foi então solicitado consentimento ao doente para terapêutica com eletroconvulsivoterapia (ECT). Realizou então oito sessões de ECT na fase aguda, sendo que após a segunda sessão já apresentava melhoria clínica, alimentando-se sozinho. Apresentou melhoria clínica progressiva, com remissão praticamente total do seu quadro clínico. Após alta retomou por completo a sua atividade profissional, mantendo a medicação anterior iniciada durante o internamento. $\bigcirc$ doente foi então seguido em consulta externa. Cerca de mês e meio depois apresentou agravamento clínico, com recidiva do seu quadro clínico inicial, tendo retomado então tratamento com ECT. Optou-se por efetuar terapêutica com ECT continuação/manutenção, inicialmente uma sessão por semana, sendo que progressivamente o período de tempo entre as sessões foi alargado para o tempo atual de uma sessão mensal. Presentemente mantém a remissão total do quadro clínico, sendo que já completou doze sessões de ECT C/M (cerca de um ano de follow-up) e mantém a terapêutica psicofarmacológica descrita.

\section{DISCUSSÃO}

O caso clínico apresentado ilustra a particularidade de um quadro depressivo grave em idoso, sem antecedentes psiquiátricos relevantes. Quadro caracterizado por humor depressivo, sintomas físicos exuberantes (emagrecimento, obstipação e disfagia) e ideação delirante congruente com o humor. Os sintomas físicos iniciaram-se após a sintomatologia depressiva. Neste caso salienta-se a verosimilhança das queixas físicas apresentadas, que obrigaram à realização de completo estudo orgânico, com exames complementares de diagnóstico para exclusão de causa médica explicativa. Do ponto de vista diagnóstico, o quadro psiquiátrico apresentado de novo em idoso, sem antecedentes psiquiátricos relevantes, obriga à exclusão de causa orgânica, nomeadamente, demência, acidentes cerebrovasculares, neoplasias, entre outras. Também importava excluir intoxicações por drogas e efeitos adversos de medicamentos.

A utilização da ECT em doentes psiquiátricos é hoje uma prática comum, verificando-se um consenso sobre a sua segurança e elevada eficácia baseada em múltiplos estudos. ${ }^{8}$ Todavia esta intervenção terapêutica permanece controversa e estigmatizada junto da população geral por informação errada ou incorreta, nomeadamente como o tratamento é hoje realizado.? A principal indicação para a ECT é a depressão major grave, sendo uma terapêutica particularmente eficaz, de rápida ação e segura na depressão geriátrica com sintomas psicóticos. ${ }^{10}$ A ECT de continuação (até aos 6 meses) e manutenção (após os 6 meses) visa prevenir respetivamente a recidiva e recorrência após remissão com sucesso, usando ECT na fase aguda. Tal como no caso apresentado, a sua utilização fica reservada para doentes que responderam com sucesso a um curso de tratamento agudo e cuja farmacoterapia de continuação e manutenção apenas faIhou na prevenção da recidiva e/ou recorrência. ${ }^{11}$

A escolha da venlafaxina prende-se com o facto de ser antidepressivo com dupla ação noradrenérgica e serotoninérgica, com indicação e comprovada eficácia na depressão major. ${ }^{12}$ Procurou-se evitar o uso de antidepressivos tricíclicos, pelo efeito adverso comum anticolinérgico, com consequente agravamento da obstipação, queixa física significativa no doente em questão. A escoIha do antipsicótico risperidona decorreu do seu perfil atípico, com poucos efeitos extrapiramidais e indicado na depressão refratária e com sintomas psicóticos. ${ }^{13} \bigcirc$ antidepressivo mirtazapina foi usado com o objetivo fundamental de potenciar a terapêutica antidepressiva e com o seu efeito sedativo ajudar a corrigir as alterações de sono. ${ }^{14,15}$

\section{CONCLUSÃO}

A peculiaridade deste caso reside na exuberância dos sintomas físicos em contexto de perturbação depressiva em doente idoso, grupo etário cujo rápido crescimento exige respostas particulares e eficazes. Uma adequada avaliação do ponto de vista orgânico e psiquiátrico permitiu um diagnóstico diferencial correto. A escolha da terapêutica com ECT em fase aguda e de continuação/ manutenção garantiu o êxito da remissão total da sintomatologia, com retomar do funcionamento social e profissional do doente.

CONFLITOS DE INTERESSE: Os autores declaram a inexistência de conflitos de interesse na realização do presente trabalho. 
FONTES DE FINANCIAMENTO: Não existiram fontes externas de financiamento para a realização deste artigo.

CONFIDENCIALIDADE DOS DADOS: Os autores declaram ter seguido os protocolos do seu centro de trabaIho acerca da publicação dos dados de doentes.

\section{REFERÊNCIAS}

1. Faculdade de Ciências Médicas da Universidade de Lisboa. Estudo Epidemiológico Nacional de Saúde Mental. 1o Relatório. Lisboa:FCMUL;2013.

2. Kendler KS, Gardner CO, Prescott CA. Toward a comprehensive developmental model for major depression in men. Am J Psychiatry. 2006;163:115-24.

3. Rot MAH, Mathew SJ, Charney DS. Neurobiological mechanisms in major depressive disorder. CMAJ. 2009;180:305-13.

4. Alexopoulos GS. Depression in the elderly. Lancet. 2005;365:1961-70.

5. Reynolds CF, Dew MA, Frank E, Begley AE, Miller MD, Cornes $C$, et al. Effects of age at onset of first lifetime episode of recurrent major depression on treatment response and illness course in elderly patients. Am J Psychiatry. 1998;155:795-9.

6. Pinquart M, Duberstein PR, Lyness JM. Treatments for later-life depressive conditions: A meta-analytic comparison of pharmacotherapy and psychotherapy. Am J Psychiatry. 2006;163:1493-501.
7. Van Der Wurff FB, Stek ML, Hoogendijk WJG, Beekman ATF. The efficacy and safety of ECT in depressed older adults: A literature review. Int J Geriatr Psychiatry. 2003;18:894-904.

8. Lisanby SH. Electroconvulsive therapy for depression. N Engl J Med. 2007;357:1939-45.

9. Kellner $\mathrm{CH}$, Greenberg RM, Murrough JW, Bryson EO, Briggs MC, Pasculli RM. ECT in treatment-resistant depression. Am J Psychiatry. 2012;169:1238-44.

10. Husain MM, Rush AJ, Fink M, Knapp R, Petrides G, Rummans $\mathrm{T}$, et al. Speed of response and remission in major depressive disorder with acute electroconvulsive therapy (ECT): A consortium for research in ECT (CORE) report. J Clin Psychiatry. 2004;65:485-91.

11. Nordenskjöld A, von Knorring L, Ljung T, Carlborg A, Brus $\mathrm{O}$, Engström I. Continuation electroconvulsive therapy with pharmacotherapy versus pharmacotherapy alone for prevention of relapse of depression: a randomized controlled trial. J ECT. 2013;29:86-92.

12. Schueler YB, Koesters M, Wieseler B, Grouven U, Kromp M, Kerekes MF, et al. A systematic review of duloxetine and venlafaxine in major depression, including unpublished data. Acta Psychiatrica Scand. 2011;123:247-65.

13. Uçok A, Gaebel W. Side effects of atypical antipsychotics: a brief overview. World Psychiatry. 2008;7:58-62.

14. Taylor D C, Kapur S. The Maudsley Prescribing Guidelines in Psychiatry. 12th ed. Oxford: Wiley-Blackwell; 2015.

15. Watanabe N, Omori IM, Nakagawa A, Cipriani A, Barbui C, Churchill R, et al. Mirtazapine versus other antidepressive agents for depression. Cochrane Database Syst Rev. 2011;12:CD006528 